\title{
Plant lipidomics based on hydrophilic interaction chromatography coupled to ion trap time-of-flight mass spectrometry
}

\author{
Yozo Okazaki • Yukiko Kamide • \\ Masami Yokota Hirai · Kazuki Saito
}

Received: 16 February 2011/ Accepted: 12 May 2011/Published online: 31 May 2011

(C) The Author(s) 2011. This article is published with open access at Springerlink.com

\begin{abstract}
Plants synthesize a wide range of hydrophobic compounds, generally known as lipids. Here, we report an application of liquid chromatography ion trap time-offlight mass spectrometry (LC-IT-TOF-MS) for plant lipidomics. Using hydrophilic interaction chromatography (HILIC) for class separation, typical membrane lipids including glycerolipids, steryl glucosides and glucosylceramides, and hydrophobic plant secondary metabolites such as saponins were analyzed simultaneously. By this method, we annotated approximately 100 molecules from Arabidopsis thaliana. To demonstrate the application of this method to biological study, we analyzed Arabidopsis mutant trigalactosyldiacylglycerol3 (tgd3), which has a complex metabolic phenotype including the accumulation of unusual forms of galactolipids. Lipid profiling by LCMS revealed that $\operatorname{tgd} 3$ accumulated an unusual form of digalactosyldiacylglycerol, annotated as $\operatorname{Gal}(\beta 1 \rightarrow 6) \beta$ GalDG. The compositional difference between normal and unusual forms of digalactosyldiacylglycerol was detected by this method. In addition, we analyzed well-known Arabidopsis mutants ats 1-1, fad6-1, and fad7-2, which are also disrupted
\end{abstract}

Electronic supplementary material The online version of this article (doi:10.1007/s11306-011-0318-z) contains supplementary material, which is available to authorized users.

Y. Okazaki $(\bowtie) \cdot$ Y. Kamide · M. Y. Hirai · K. Saito RIKEN Plant Science Center, Tsurumi-ku,

Yokohama 230-0045, Japan

e-mail: okazaki@psc.riken.jp

M. Y. Hirai

Japan Science and Technology Agency, CREST, Tokyo, Japan

K. Saito

Graduate School of Pharmaceutical Sciences, Chiba University, Inage-ku, Chiba 263-8522, Japan in lipid metabolic genes. Untargeted lipidome analysis coupled with multivariate analysis clearly discriminated the mutants and their distinctive metabolites. These results indicated that HILIC-MS is an efficient method for plant lipidomics.

Keywords Hydrophilic interaction chromatography . Ion trap time-of-flight mass spectrometry · Lipidome . Lipidomics

\section{Introduction}

Recent developments in mass spectrometry make it feasible to analyze metabolome. The metabolome represents the ultimate cell phenotype, which is controlled through the transmission of genetic information. Thus, one must analyze a snapshot of the metabolome to precisely understand cell status. However, plants synthesize a huge array of metabolites that cannot be revealed by any single methodology (Saito and Matsuda 2010). Plant lipidomics based on tandem mass spectrometry using a triple quadrupole mass spectrometer is frequently applied to the analyses of several polar glycerolipids and sphingolipids using highly selective functions including precursor ion and neutral loss scanning or multiple reaction monitoring according to well-characterized mass fragmentation patterns (Brügger et al. 1997; Welti et al. 2003; Welti and Wang 2004; Markham and Jaworski 2007). These approaches have revealed many new insights into plant lipid metabolism and its genetic foundations (Chen et al. 2008; Beaudoin et al. 2009; Jao et al. 2009; Michaelson et al. 2009; Zhang et al. 2009; Peters et al. 2010; Roudier et al. 2010; Xiao et al. 2010). However, plants synthesize lipids that are not yet classified or well characterized by mass spectrometry. Focused metabolomics 
approaches cannot address these lipids. An improved analytical method appropriate for a wide range of plant lipid classes and lipidome can facilitate future studies on plant lipid metabolism and our understanding of the diversity of plant metabolome.

In this study, liquid chromatography ion trap time-offlight mass spectrometry (IT-TOF-MS) was used to analyze the plant lipidome; high resolution $\mathrm{MS}^{\mathrm{n}}$ was expected to be an efficient method to characterize the complex mixture of plant metabolites (Shibano et al. 2007; Zhou et al. 2009; Aboshi et al. 2010; Liu et al. 2010). The power of $M^{n}$ for metabolite profiling can be enhanced by combination with chromatographic separation. Since many lipid classes consist of hydrophobic moieties (i.e., fatty acid and ceramide) and polar functional groups (phosphate, glucose, and galactose), normal phase chromatography provides efficient class separation. We employed hydrophilic interaction chromatography (HILIC), which is often used to analyze polar compounds. A similar analytical system was used for targeted profiling of polar glycerolipids of mutant plants (Okazaki et al. 2009). We expanded the coverage of this analytical system to other major membrane lipid classes and lipophilic plant secondary metabolites. In addition, we applied our method to the analysis of the lipidome of several A. thaliana mutants.

For a case study of the biological application of our method, we analyzed the Arabidopsis tgd3 mutant (trigalactosyldiacylglycerol3) (Fig. 1) (Lu et al. 2007). The biosynthesis of lipids occurs in the discrete subcellular biogenic membranes of different cellular compartments.

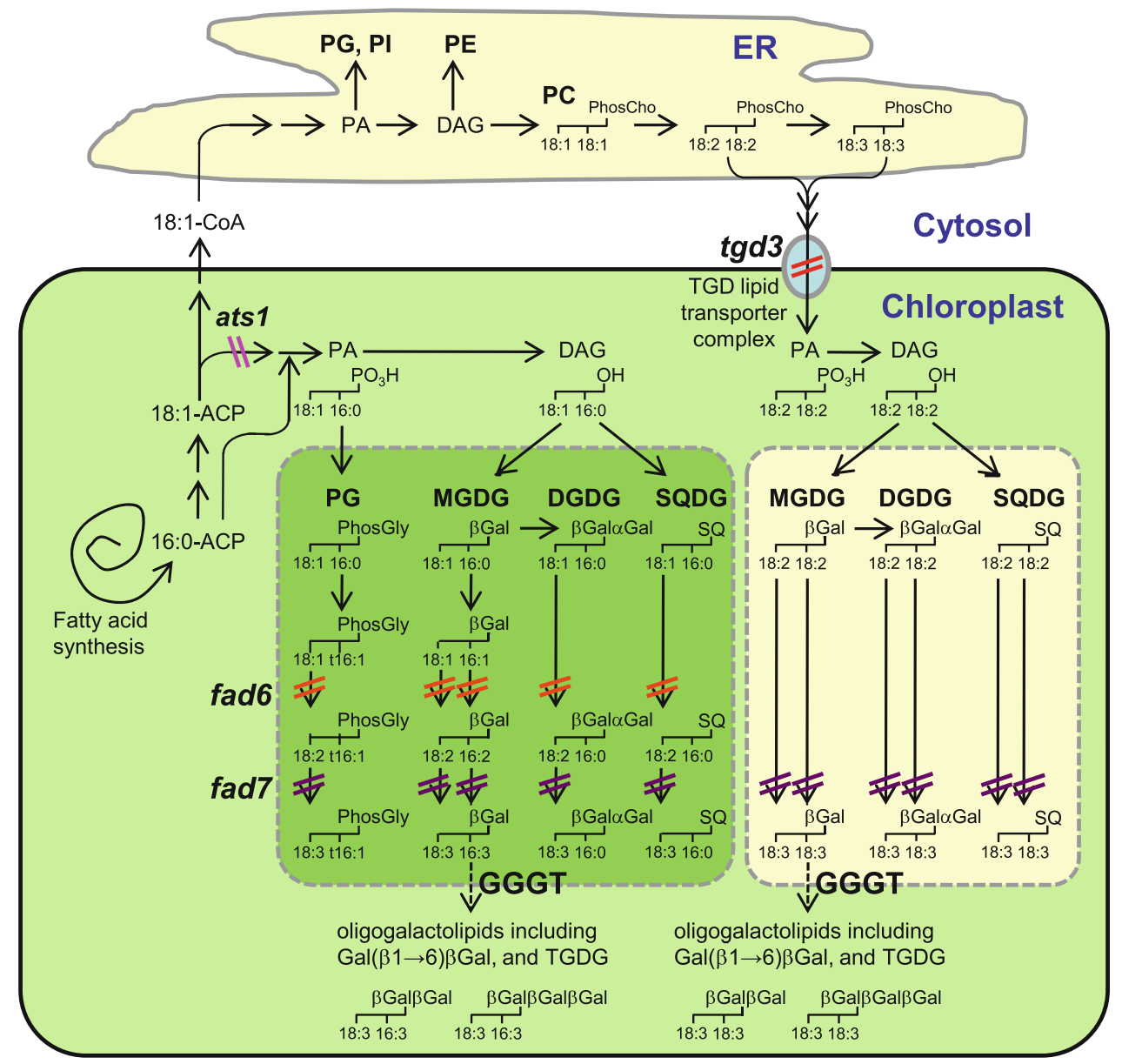

Fig. 1 Glycerolipid biosynthesis in A. thaliana leave. An abbreviated biosynthetic scheme for the major plant glycerolipids is represented. Breaks in the pathway represent the mutation sites described in case studies 1 and 2. The set of reactions occurring within the chloroplast are termed the "prokaryotic pathway" and those that involve glycerolipid synthesis in the ER and subsequent transfer to the chloroplast constitute the "eukaryotic pathway". Dashed arrows indicate the enhanced biosynthetic pathway of oligogalactolipids in the $\operatorname{tgd} 3$ mutant. For simplification, another pathway to transport ER- derived glycerolipid to chloroplasts, mediated by lipin-like phosphatases, is omitted (Nakamura et al. 2009). This metabolic pathway is illustrated according to Somerville et al. (Somerville et al. 2000) with modifications. $A C P$ acyl carrier protein, $P A$ phosphatidic acid, $D A G$ diacylglycerol, PhosCho phosphocholine, PhosGly phosphoglycerol, t16:1 hexadec-3-trans-enoic acid, $S Q$ sulfoquinovose, $G G G T$ galactolipid:galactolipid galactosyltransferase, $\beta$ Gal $\beta$ Gal $\operatorname{Gal}(\beta 1 \rightarrow 6) \beta \varpi$ Gal, $\beta$ Gal $\alpha$ Gal Gal $(\beta 1 \rightarrow 6) \alpha \mathrm{Gal}$ 
Therefore, the intracellular trafficking of lipids is essential for the assembly of membrane systems. In seed plants, the quantitatively largest flux of lipid precursors is between the 2 major sites of glycerolipid assembly, namely the endoplasmic reticulum (ER) and the plastid envelopes (Somerville and Browse 1996; Benning 2009). These biogenic membranes cooperate and constitute parallel pathways for thylakoid lipid biosynthesis in the ER and the plastid. Recently, mutants of Arabidopsis lacking ER-derived thylakoid lipid species were isolated and four proteins involved in ER-to-plastid lipid transport were identified (Xu et al. 2003; Xu et al. 2005; Awai et al. 2006; Lu et al. 2007; $\mathrm{Xu}$ et al. 2008). These loci were named TRIGALACTOSYLDIACYLGLYCEROL (TGD) because of the accumulation of the new galactoglycerolipid trigalactosyldiacylglycerol (TGDG). Based on detailed analyses of the mutants and the respective proteins, it was proposed that TGD 1, 2 and 3 form a complex in the inner chloroplast envelope membrane responsible for delivering ER-derived phosphatidate (PA) to the inside of the inner envelope membrane. PA dephosphorylation gives rise to DAG, which is used for galactolipid biosynthesis in plastid envelope membranes. The fourth protein, TGD4, is involved in lipid transport between the ER and outer chloroplast envelope membrane (Xu et al. 2008). In addition to TGDG, Arabidopsis mutants deficient in TDG1 accumulate an unusual form of digalactosyldiacylglycerol (DGDG), $\operatorname{Gal}(\beta 1 \rightarrow 6) \beta \mathrm{GalDG}$. NMR analysis of purified DGDG revealed that DGDG in $\operatorname{tgdl}$ was a mixture of $\operatorname{Gal}(\beta 1 \rightarrow 6) \beta \mathrm{GalDG}$ and normal DGDG $(\operatorname{Gal}(\alpha 1 \rightarrow 6) \beta$ GalDG), but the detailed profile of $\operatorname{Gal}(\beta 1 \rightarrow 6) \beta \mathrm{GalDG}$ in tgd mutants remains unclear $(\mathrm{Xu}$ et al. 2003). Since all the TGD proteins are involved in the same metabolic function, $\operatorname{tgd} 3$ was also expected to accumulate $\operatorname{Gal}(\beta 1 \rightarrow 6) \beta \mathrm{GalDG}$, although there has been no detailed analysis of intact lipids in $\operatorname{tgd} 3$. We analyzed DGDG in $\operatorname{tgd} 3$ to evaluate the separation ability and coverage of our method, and generated a detailed profile of two forms of intact DGDG in this mutant.

We also analyzed several well-characterized Arabidopsis mutants (ats1-1, fad6-1, and fad7-2) which were used for studies on the biosynthesis of polyunsaturated fatty acids and plastidic membrane lipids (Fig. 1) (Kunst et al. 1988; Iba et al. 1993; Falcone et al. 1994; Xu et al. 2006), to determine whether untargeted lipidome analysis of these mutants could reproduce the results of these previous reports.

\section{Materials and methods}

\subsection{Chemicals}

1,2-Dioctanoyl-sn-glycero-3-phosphocholine

(10:0/10:0

PC) and $30 \%$ ammonium hydroxide (A.C.S. reagent) were purchased from Sigma-Aldrich (St. Louis, MO. USA). Other phospholipid standards (PC, PE, PG, PA, and PI) and a soybean glucosylceramide [comprised primarily ( $>98 \%$ ) of ceramide with 4,8-sphingadiene and $\alpha$-hydroxypalmitic acid] were obtained from Avanti Polar Lipids (Alabaster, AL, USA). Steryl glucoside and tomatine were obtained from Larodan Fine Chemicals (Malmö, Sweden) and Tokyo Chemical Industry (Tokyo, Japan). Solvents for LCMS and other chemicals were purchased from Wako Pure Chemical Industries (Osaka, Japan) unless stated otherwise.

\subsection{Plant materials}

Seeds of $A$. thaliana mutants (SALK_040335 for tgd3, CS200 for ats 1-1, CS207 for fad6-1, and CS8042 for fad7-2) were obtained from the ABRC (Ohio, USA). The T-DNA insertion site in $\operatorname{tgd} 3$ was confirmed by sequencing of PCR fragments at the left border of the T-DNA amplified using LBa1 (5'-TGGTTCACGTAGTGGGCCATCG-3') and gene-specific primer (5'-TGCTTCAGTGGTATGTCATGT GAAAGTAT- $3^{\prime}$ ). Arabidopsis plants were grown on agarsolidified Murashige-Skoog medium containing $1 \%(\mathrm{w} / \mathrm{v})$ sucrose and $\mathrm{MS}$ vitamin at $22^{\circ} \mathrm{C}$ under a 16-h-light/8-h-dark cycle for 18 days. Plant tissues were harvested $6 \mathrm{~h}$ after the onset of the light phase, frozen in liquid nitrogen, and stored at $-80^{\circ} \mathrm{C}$ until lipid extraction. Oat seeds (Avena strigosa cv. Hayoats) were purchased from Snow Brand Seed (Sapporo, Japan), and rice seeds (Oryza sativa L. cv. Nipponbare) were the kind gift of Prof. Masahiro Yano at the National Institute of Agrobiological Sciences. Caryopses of rice and oat were incubated for 5 days under the same growth conditions used for A. thaliana.

\subsection{Sample preparation}

Total lipids were extracted according to the method of Bligh and Dyer (Bligh and Dyer 1959) with slight modifications. The frozen plant material was milled on a Mixer Mill MM300 (Retsch, Haan, Germany) for $2 \mathrm{~min}$ at $15 \mathrm{~Hz}$ with a zirconium bead. The milling process was carried out under cryogenic conditions (the grinding jars were cooled with liquid nitrogen before each milling process to ensure a low milling temperature to keep the plants frozen). The frozen powdered plant material was kept in liquid nitrogen and a 20-fold volume of extraction solvent $\left(\mathrm{CHCl}_{3}-\right.$ $\mathrm{MeOH}-\mathrm{H}_{2} \mathrm{O}=50: 100: 31.45, \mathrm{v} / \mathrm{v} / \mathrm{v}$ ) was added with $1 \mu \mathrm{M}$ 10:0/10:0 PC as the internal standard, followed by vigorous stirring on a vortex mixer. After dark incubation for $5 \mathrm{~min}$ at room temperature, the homogenate was centrifuged at $3,000 \times g$ for $3 \mathrm{~min}$ at room temperature. Two hundred microliters of supernatant was mixed with $\mathrm{CHCl}_{3}$ and $\mathrm{H}_{2} \mathrm{O}$ (52.6 $\mu \mathrm{l}$ each), stirred on a vortex mixer, and incubated on 
ice for $15 \mathrm{~min}$ in darkness. The mixture was centrifuged at $1,000 \times g$ for $3 \mathrm{~min}$, and $85 \mu \mathrm{l}$ of the lower layer was collected. The organic phase was dried by a centrifugal concentrator at room temperature and the residue was dissolved in $81 \mu \mathrm{l}$ of $\mathrm{CHCl}_{3}$ for LC-MS analysis.

\subsection{Mass spectrometry and data analysis}

Mass spectra were recorded on an LCMS-IT-TOF mass spectrometer combined with an LC-20AD HPLC system (Shimadzu Corporation, Kyoto, Japan) using an Atlantis HILIC silica column (average particle size, $3 \mu \mathrm{m} ; 2.1 \mathrm{~mm}$ i.d., $100 \mathrm{~mm}$ long; Waters, MA, USA) as reported previously with some alterations (Okazaki et al. 2009). A two-solvent system was used to generate the mobile phase: solvent A, methanol-water $(95: 5, \mathrm{v} / \mathrm{v})$ containing $0.2 \%$ ammonium formate $(\mathrm{pH} 5.9)$; solvent $\mathrm{B}$, acetonitrile-methanol-water $(95: 2: 3, \mathrm{v} / \mathrm{v} / \mathrm{v})$ containing $0.2 \%$ ammonium formate ( $\mathrm{pH}$ 5.9). The $\mathrm{pH}$ of both solvents $\mathrm{A}$ and $\mathrm{B}$ was adjusted by adding $30 \% \mathrm{NH}_{4} \mathrm{OH}$ to the mixtures of solvents containing $0.2 \%(\mathrm{v} / \mathrm{v})$ formic acid. The gradient conditions for lipid separation were as follows: $100 \%$ solvent B $(0-3.33 \mathrm{~min}), \quad 100-65 \%$ solvent B (3.33-10 min), 65-30\% (10-11.33 min), 30\% solvent B (11.33-14.66 min), $100 \%$ solvent B (14.66-40 min). The flow rate was held at $0.2 \mathrm{ml} \mathrm{min}^{-1}$ increased to $0.4 \mathrm{ml} \mathrm{min}{ }^{-1} 14.66 \mathrm{~min}$ after gradient initiation, maintained for $13.33 \mathrm{~min}$, and then decreased to $0.2 \mathrm{ml} \mathrm{min}^{-1}$. Total elution time was $40 \mathrm{~min}$. The column temperature was $25^{\circ} \mathrm{C}$.

Peak picking and peak alignment were performed by Profiling Solution (version 1.0.76.0) (Shimadzu Corporation, Kyoto, Japan). The Profiling Solution parameters were as follows: ion $\mathrm{m} / \mathrm{z}$ tolerance, $20 \mathrm{mDa}$; Ion RT tolerance, $0.1 \mathrm{~min}$; Ion intensity threshold, 2e4; detect isomer valley, 20\%; allow some ion without isotope peak, ON; time range for processing, 0-20 min. The data matrix containing 534 ions was exported from Profiling Solution and normalized based on the intensity of $\left[\mathrm{M}+\mathrm{HCO}_{2}\right]^{-}$of 10:0/10:0 PC. Peak height of individual galactolipid molecules were calculated based on the $m / z$ values of $\left[\mathrm{M}+\mathrm{HCO}_{2}\right]^{-}$. Because lipid species with the same polar head eluted at almost the same retention time, corrections for overlap of isotopic variants in higher-mass lipids were applied. Annotation was based on theoretical $\mathrm{m} / \mathrm{z}$ values of each possible glycerolipid species in plants and retention time of authentic compounds with same polar head using an in-house Perl script. After this process, data was pareto-scaled and subjected to PCA and OPLS-DA using SIMCA-P (version 11.0.0.0, Umetrics, Umeä, Sweden). LC-MS data and metadata used in case study 2 are available as Supplementary Material 1.

\section{Results and discussion}

3.1 HILIC-MS can be used to analyze various membrane lipids and lipophilic secondary metabolites

Crude lipid extracts of leaves and roots of the model plant A. thaliana was analyzed by HILIC coupled to IT-TOFMS. As reported previously, typical membrane glycerolipids including MGDG, DGDG, SQDG, PC, PE, PG, and PI were detected in leaves and roots based on the authentic compounds (Fig. 2) (Okazaki et al. 2009). Similar lipid profiles were observed in the leaves of rice (Oryza sativa), an important cereal plant (Supplementary Fig. 1). Since HILIC is a version of normal phase chromatography, lipid molecules with the same polar head group had similar retention times (Malavolta et al. 2004; Houjou et al. 2005; Taguchi et al. 2007). With these properties, HILIC is similar to silica gel chromatography. However, the chromatography solvents were different: in our method, we used a mixture of methanol, acetonitrile, and water, while ordinary silica gel chromatography for plant lipid analysis is often performed using chloroform (Sugawara and Miyazawa 1999; Malavolta et al. 2004; Pacetti et al. 2007;

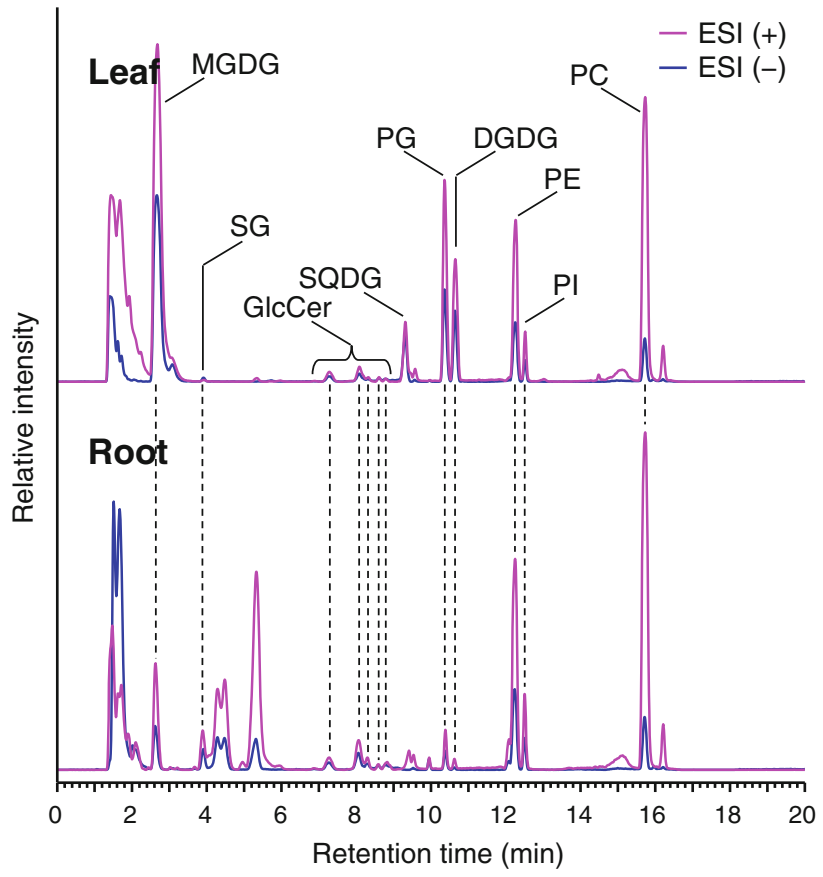

Fig. 2 Total ion current chromatograms of lipids from A. thaliana leaves and roots. Total ion currents represent the compositional difference in lipid classes between leaves and roots of A. thaliana. The baseline is shifted for convenience. The following lipid classes were observed in both tissues: $M G D G$ monogalactosyldiacylglycerol, $S G$ steryl glucoside, GlcCer glucosylceramide, $P G$ phosphatidylglycerol, $D G D G$ digalactosyldiacylglycerol, $P E$ phosphatidylethanolamine, $P I$ phosphatidylinositol, and $P C$ phosphatidylcholine 
Yunoki et al. 2009; Rocha et al. 2010). Thus, lipid analysis by HILIC can reduce toxic halogenated solvent waste and is better suited for electrospray ionization, the most normal ionization mode of LC-MS.

\subsection{Annotation of nonglycerolipid component in A. thaliana}

As shown in Fig. 2, there are many signals not attributable to typical polar glycerolipids. To determine what other lipids kinds of can be analyzed by this method, commercially available compounds were subjected to LC-MS, and steryl glucoside (SG) and glucosylceramide (GlcCer) were newly found to show adequate retention on HILIC. These lipids are major nonglycerolipid components of the cellular membrane. To analyze the diverse molecular species belonging to these lipid classes, their mass spectra were analyzed. Since the authentic SG compound was derived from soybean, not chemically synthesized, it contained several SG species that also could be detected in A. thaliana. Based on their mass spectra, the SG species in A. thaliana were annotated as glucosides of sitosterol, campesterol, and stigmasterol (Table 1). To obtain the fragmentation pattern of GlcCer, we analyzed glucosylceramide consisting of 4,8sphingadiene and $\alpha$-hydroxypalmitic acid. When this compound was analyzed by LC-MS, $[\mathrm{M}+\mathrm{H}]^{+}$and $[\mathrm{M}+\mathrm{H}$ - glucose $(=180 \mathrm{Da})]^{+}$were clearly observed at $\mathrm{m} / \mathrm{z}$ 714.552 and $\mathrm{m} / \mathrm{z} 534.489$ (Fig. 3a). The MS/MS of $[\mathrm{M}+\mathrm{H}]^{+}$attributed the fragment at $m / z, 262.252$ to 4,8 sphingadiene, the long-chain-base (LCB) of this GlcCer molecule (Fig. 3b). According to this fragmentation pattern, GlcCer species of A. thaliana leaves were annotated (Table 1). The result was in accordance with the previous report (Markham and Jaworski 2007). When the GlcCer composition of $A$. thaliana was investigated based on
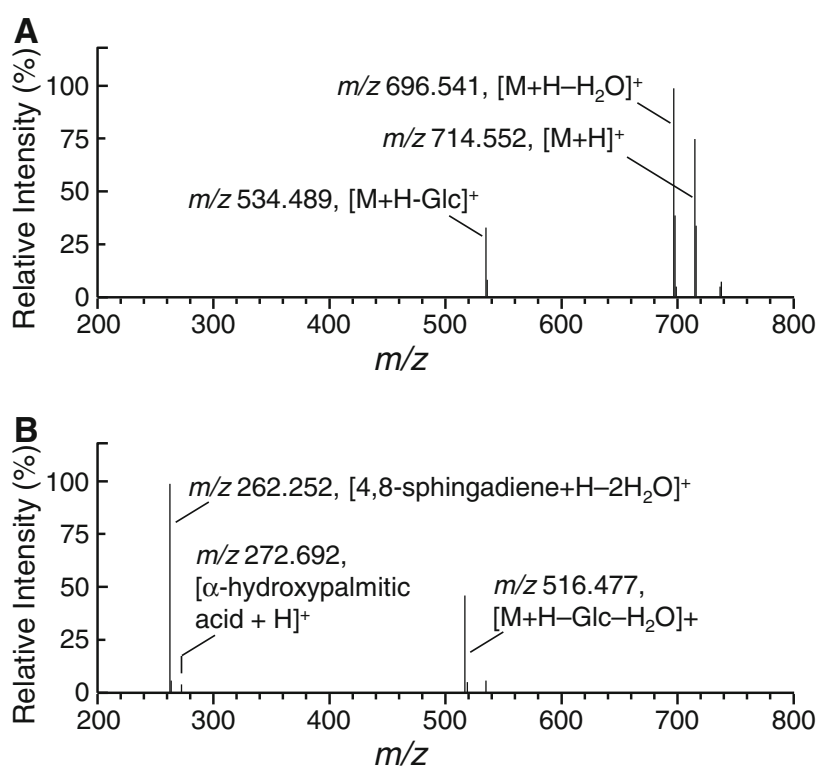

Fig. 3 Analysis of GlcCer by IT-TOF-MS. a Positive MS and b MS/ MS of glucosylceramide consisting of 4,8-sphingadiene and $\alpha$-hydroxypalmitic acid are displayed (precursor ion, $[\mathrm{M}+\mathrm{H}]^{+}$)

extracted ion chromatograms (XICs), 2 peaks were observed for the mass-to-charge ratio of some GlcCer species (Fig. 4), suggesting that these ions were isomers which have $E$ or $Z$ configurations at double bonds in the LCB moiety (Sperling and Heinz 2003; Markham et al. 2006). In addition to these polar membrane lipids that are universally detected in various plant species, lipophilic plant secondary metabolites also could be analyzed. Tomatine, a glycoalkaloid saponin of tomato (Solanum lycopersicum), exhibited adequate retention $\left(t_{\mathrm{R}} 14.1 \mathrm{~min}\right)$. In addition, triterpenoid saponin avenacin A-1 was detected ( $\left.t_{\mathrm{R}} 12.8 \mathrm{~min}\right)$ in lipid extracts from the roots of diploid oat (Avena strigosa). These saponins have very important roles in plants as defense

Table 1 Steryl glucosides and glucosylceramides detected in A. thaliana

\begin{tabular}{llll}
\hline Annotation & $t_{\mathrm{R}}(\mathrm{min})$ & $\mathrm{MS}(\mathrm{m} / \mathrm{z})$ & $\mathrm{MS} / \mathrm{MS}(\mathrm{m} / z)$ \\
\hline Sitosteryl glucoside & 3.9 & $621.433^{\mathrm{a}}, 611.404^{\mathrm{b}}$ & \\
Stigmasteryl glucoside & 3.9 & $619.415^{\mathrm{a}}, 609.304^{\mathrm{b}}$ & $272.257^{\mathrm{e}}, 264.269^{\mathrm{f}}$ \\
Campesteryl glucoside & 4.0 & $607.416^{\mathrm{a}}$ & $272.256^{\mathrm{e}}, 280.254^{\mathrm{f}}, 262.243^{\mathrm{g}}$ \\
$\mathrm{d} 18: 1 / \mathrm{h} 16: 0 \mathrm{GlcCer}$ & $7.1,7.5$ & $716.565^{\mathrm{c}}, 554.518^{\mathrm{d}}$ & $262.245^{\mathrm{g}}$ \\
$\mathrm{t} 18: 1 / \mathrm{h} 16: 0 \mathrm{GlcCer}$ & $8.5,8.9$ & $732.560^{\mathrm{c}}, 570.509^{\mathrm{d}}$ & $262.242^{\mathrm{g}}$ \\
$\mathrm{t} 18: 1 / \mathrm{h} 22: 0 \mathrm{GlcCer}$ & $8.1,8.3$ & $816.651^{\mathrm{c}}, 654.607^{\mathrm{d}}$ & $262.243^{\mathrm{g}}$ \\
$\mathrm{t} 18: 1 / \mathrm{h} 24: 1 \mathrm{GlcCer}$ & $7.9,8.2$ & $842.667^{\mathrm{c}}, 680.623^{\mathrm{d}}$ & \\
$\mathrm{t} 18: 1 / \mathrm{h} 24: 0 \mathrm{GlcCer}$ & $7.9,8.1$ & $844.687^{\mathrm{c}}, 682.637^{\mathrm{d}}$ & $262.246^{\mathrm{g}}$ \\
$\mathrm{t} 18: 1 / \mathrm{h} 26: 1 \mathrm{GlcCer}$ & 8.0 & $870.695^{\mathrm{c}}$ & $872.711^{\mathrm{c}}, 710.667^{\mathrm{d}}$ \\
$\mathrm{t} 18: 1 / \mathrm{h} 26: 0 \mathrm{GlcCer}$ & 7.8 & & \\
\hline
\end{tabular}

Superscripts indicate the assignment of each ion: $\mathrm{a}, \mathrm{b}, \mathrm{c}, \mathrm{d}, \mathrm{e}, \mathrm{f}$, and g are corresponding to $\left[\mathrm{M}+\mathrm{HCO}_{2}\right]^{-},[\mathrm{M}+\mathrm{Cl}]^{-},[\mathrm{M}+\mathrm{H}]^{+},[\mathrm{M}+\mathrm{H}-$ glucose $]^{+}$, [hydroxyfatty acid amide $\left.+\mathrm{H}\right]^{+},\left[\mathrm{LCB}+\mathrm{H}-2 \mathrm{H}_{2} \mathrm{O}\right]^{+},\left[\mathrm{LCB}+\mathrm{H}-3 \mathrm{H}_{2} \mathrm{O}\right]^{+}$. LCB and fatty acid nomenclature are as follows, $\mathrm{d} / \mathrm{t}$ (di/trihydroxy) 18 (18 carbon chain):1 (one desaturation) followed by fatty acid; c/h (non-hydroxy/hydroxy) 12 (12 carbon chain):0 (no desaturation) 


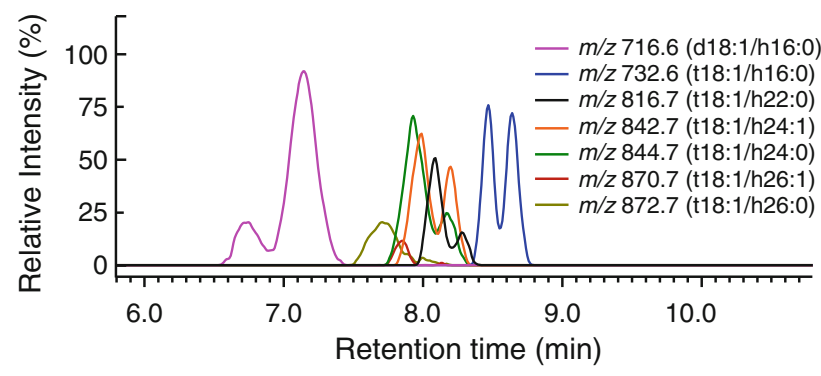

Fig. 4 Analysis of GlcCer molecular profiles. Extracted ion chromatograms (XICs) for $m / z$ values of $[\mathrm{M}+\mathrm{H}]^{+}$are displayed to compare GlcCer molecular profiles in A. thaliana

compounds against pathogens (Papadopoulou et al. 1999; Friedman 2002), and similar compounds are widely distributed throughout the plant kingdom (Connolly and Hill 2010). Since MS/MS of these compounds were in accordance with their chemical structures (Supplementary Fig. 2), similar compounds could be analyzed by this method. These data indicated that our method could be applied to the analyses of a wide range of plant lipids including glycerolipids, steryl glucosides, glucosylceramides, and lipidic secondary metabolites. In the negative ion mode, we detected approximately 500 lipid signals from A. thaliana leaves and annotated 96 molecules as shown in Supplementary Table 1 . The relative standard deviation of retention time of internal standard (10:0/10:0 PC) was 6.6\% $(n=16)$, and the averaged $\mathrm{m} / z$ value of $[\mathrm{MH}]^{+}$of internal standard was $566.378 \pm 1.6 \mathrm{mDa}$ (calcd for $\mathrm{C}_{28} \mathrm{H}_{56} \mathrm{~N}_{8} \mathrm{O}_{8} \mathrm{P}$ : 566.3822) for the dataset shown in Supplemental Table 1. The root-extracted lipids observed at $t_{\mathrm{R}} 4 \sim 6 \mathrm{~min}$ and $t_{\mathrm{R}}$ $9 \sim 10$ min remain unidentified. However, a significant qualitative difference between the lipid profiles of leaves and roots was revealed.

\subsection{Applications of HILIC-MS for biological studies- case study 1}

We analyzed digalactosyldiacylglycerol (DGDG) in the Arabidopsis tgd3 (trigalactosyldiacylglycerol3) mutant, which cannot transport phosphatidic acid from the endoplasmic membrane (ER) to the plastid ( $\mathrm{Lu}$ et al. 2007) (Fig. 1). DGDG is a major constituent of the plastid membrane. The diacylglycerol moiety of galactoglycerolipids is synthesized de novo in the plastid or assembled at the ER and must be imported into the plastid (Roughan and Slack 1982). The head group of DGDG is characterized by a terminal $\alpha$-galactose moiety $(1 \rightarrow 6)$ linked to the inner $\beta$-galactose residue $(\mathrm{Gal}(\alpha 1 \rightarrow 6) \beta \mathrm{GalDG})$ (Benning and Ohta 2005). However, the tgdl mutant of A. thaliana and some plant species (e.g., Adzuki bean) contain an additional form of DGDG with the second galactose in the $\beta$-anomeric configuration $(\mathrm{Gal}(\beta 1 \rightarrow 6) \beta \mathrm{GalDG})($ Kojima et al. 1990;

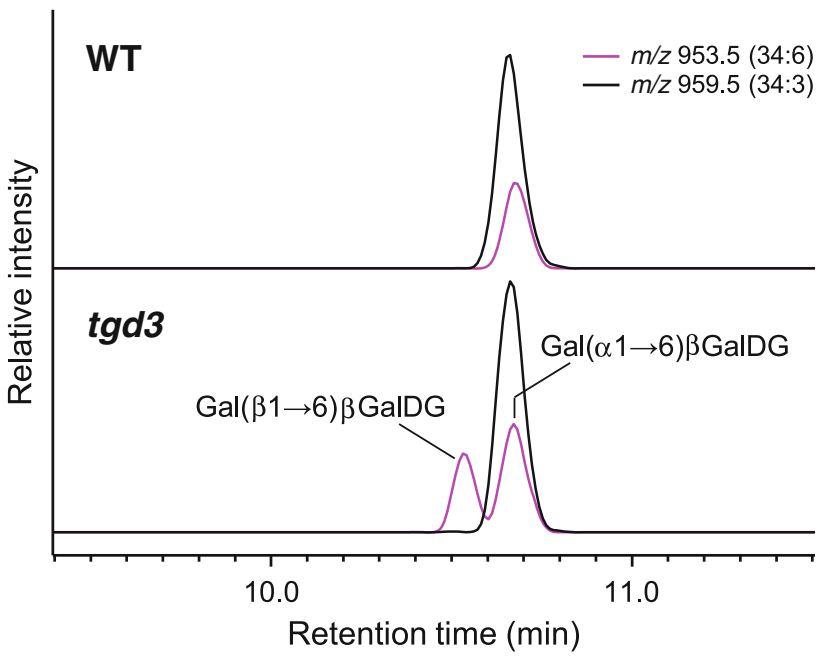

Fig. 5 Analysis of DGDG in wild type and $\operatorname{tgd} 3$ mutant of $A$. thaliana XICs of $\left[\mathrm{M}+\mathrm{HCO}_{2}\right]^{-}$display the relative levels of $34: 6$ DGDG and 34:3 DGDG molecular species in the leaves of wild type and tgd3 mutant of A. thaliana. The species labels, total acyl carbons:total acyl double bonds, are shown in parentheses. The intensity of $\left[\mathrm{M}+\mathrm{HCO}_{2}\right]^{-}$of 34:3 DGDG in the wild type is set as $100 \%$. The baseline is shifted for convenience

Xu et al. 2003; Kelly and Dormann 2004; Benning and Ohta 2005; Hölzl and Dörmann 2007). A mixture of these forms of DGDG was observed in an Arabidopsis tgdl mutant based on the signals of anomeric protons in the ${ }^{1} \mathrm{H}$ NMR spectra of purified DGDG (Xu et al. 2003), but the detailed composition of DGDG in tgd mutants remained unclear. Thus, we evaluated the separation ability and coverage of our method by applying it to the analysis of these two forms of DGDG in a $\operatorname{tg} d 3$ mutant. As reported previously (Lu et al. 2007), an accumulation of trigalactosyldiacylglycerol (TGDG) was observed (Supplementary Fig. 3). Thus, we used this experimental material to analyze this unusual form of DGDG. When the composition of DGDG in $\operatorname{tgd} 3$ was investigated based on XICs, 2 peaks were observed for the mass-to-charge ratio of a particular DGDG molecule (34:6 DGDG) (Fig. 5). Since the former-eluting isomer was detected only in the $\operatorname{tg} d 3$ mutant, this peak was annotated as $\mathrm{Gal}(\beta 1 \rightarrow 6) \beta \mathrm{GalDG}$. MS/MS of $34: 6 \mathrm{Gal}(\beta 1 \rightarrow 6) \beta \mathrm{GalDG}$ and 34:6 Gal $(\alpha 1 \rightarrow 6) \beta$ GalDG were quite similar (Supplementary Fig. 4), which supported their annotations. Based on these results, the profile of 2 forms of DGDG in $\operatorname{tgd} 3$ was analyzed (Fig. 6). The major molecular species of $\operatorname{Gal}(\beta 1 \rightarrow 6) \beta \mathrm{GalDG}$ was determined to be $34: 6$, while the major molecules of $\mathrm{Gal}(\alpha 1 \rightarrow 6) \beta \mathrm{GalDG}$ were $34: 3$ and 36:6 Gal $(\alpha 1 \rightarrow 6) \beta$ GalDG.

The normal form of DGDG, $\operatorname{Gal}(\alpha 1 \rightarrow 6) \beta \mathrm{GalDG}$, is synthesized from MGDG by UDP-galactose-dependent DGD1/2 enzymes (Dörmann et al. 1999; Kelly and Dörmann 2002), whereas $\operatorname{Gal}(\beta 1 \rightarrow 6) \beta$ GalDG is synthesized from two molecules of MGDG by galactolipid:galactolipid 


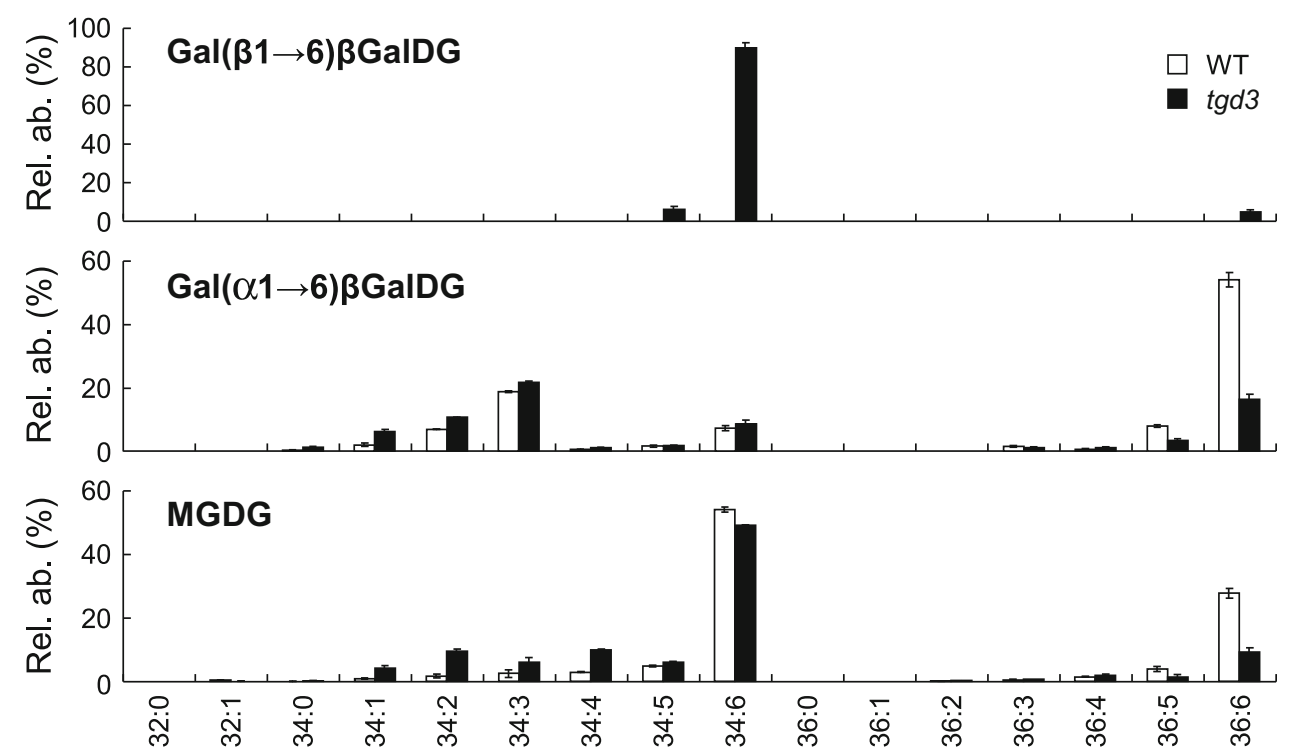

Lipid molecular species (total acyl carbons: total double bonds)

Fig. 6 Galactolipid profiles in the leaves of wild type and $\operatorname{tg} d 3$ mutant of A. thaliana. Peak heights of individual lipid molecules were determined by monitoring their molecular-related ions (details are described in the "Materials and methods" section). Levels of individual lipid molecules in the wild-type and mutant lines are expressed as relative abundance (Rel. ab. \%). In the case of $\operatorname{Gal}(\beta 1 \rightarrow 6) \beta$ GalDG, Rel. ab. (\%) is (Peak height of individual

galactosyltransferase (GGGT) (Fig. 1) (Benning and Ohta 2005). GGGT is also involved in the synthesis of oligogalactolipids including TGDG, and enhanced accumulation of unusual forms of galactolipids in tgd mutants is due to increased GGGT activity (Xu et al. 2003; Awai et al. 2006; Lu et al. 2007; Xu et al. 2008). Recently, the gene encoding GGGT was identified as SENSITVE TO FREEZING 2 (SFR2), a gene essential for freeze tolerance in Arabidopsis (Moellering et al. 2010). The molecular profile of $\operatorname{Gal}(\beta 1 \rightarrow 6) \beta$ GalDG was more similar to that of MGDG than $\operatorname{Gal}(\alpha 1 \rightarrow 6) \beta$ GalDG (Fig. 6). This result is expected, given their biosynthesis, but the molecular profile of $\operatorname{Gal}(\beta 1 \rightarrow 6) \beta$ GalDG does not exactly reflect that of MGDG; the minor molecular species found in MGDG (34:1 34:4 species) which were increased in the $\operatorname{tg} d 3$ mutant were not detected in $\operatorname{Gal}(\beta 1 \rightarrow 6) \beta$ GalDG. These data suggest that the GGGT enzyme may have greater affinity for MGDG consisting of polyunsaturated fatty acids or that the substrate-supplying mechanisms for GGGT are biased to polyunsaturated molecules. There is also the possibility that $\operatorname{Gal}(\beta 1 \rightarrow 6) \beta$ GalDG may be a better substrate than MGDG for plastidic fatty acid desaturases. Increased fatty acid unsaturation has long been correlated with enhanced freezing tolerance or adaptations to growth at low above-freezing temperatures (Steponkus 1984; Uemura et al. 1995). Recently, remodeling of the membrane lipid profile by GGGT was indicated to be lipid molecule/sum of peak heights of $\operatorname{Gal}(\beta 1 \rightarrow 6) \beta \mathrm{GalDG}$ of $\operatorname{tgd3}) \times 100$. In the case of $\mathrm{Gal}(\alpha 1 \rightarrow 6) \beta \mathrm{GalDG}$ and MGDG, Rel. ab. (\%) is (Peak height of individual lipid molecule/sum of peak heights of lipids of the same class in wild type $) \times 100$. Each data point represents the mean of 3 experiments \pm standard deviation (SD)

important for freezing tolerance in Arabidopsis (Moellering et al. 2010). Thus, the increased fatty acid unsaturation in $\operatorname{Gal}(\beta 1 \rightarrow 6) \beta$ GalDG may be expected for the lipid class synthesized by GGGT, which is required for freezing tolerance.

\subsection{Applications of HILIC-MS for biological studies - case study 2}

Lipid profiles of well characterized Arabidopsis mutants ats $1-1$, fad6-1, and fad7-2 were analyzed to examine whether untargeted lipidome analysis of these mutants could produce results consistent with those obtained by conventional analytical method which require prepurification procedure of each lipid class and chemical derivatization. The ats 1-1 mutant lacks most of its plastidic acyl-ACP:glycerol-3-phosphate acyltransferase activity (Fig. 1) and is blocked in the plastid galactoglycerolipid biosynthesis pathway (Kunst et al. 1988; Xu et al. 2006). Thus, the amount of galactolipids containing ER-derived diacylglycerol moiety increases to maintain plastidic membrane function. ER-derived glycerolipids have an 18-carbon fatty acid at $s n$-2 of glycerol, while glycerolipids synthesized de novo in plastids have a 16-carbon fatty acid at $s n-2$ (Heinz and Roughan 1983; Browse et al. 1986). Thus, the ratio of 16-carbon fatty acids to 18 -carbon fatty acids in plastid glycerolipids is reduced by disruption of 
ATS1. The fad6-1 mutant lacks the chloroplast $\omega-6$ fatty acid desaturase responsible for synthesis of 16:2 and 18:2 fatty acids in galactolipids, SQDG, and PG (Falcone et al. 1994), while fad7-2 is disrupted for the chloroplast $\omega-3$ fatty acid desaturase responsible for synthesis of 16:3 and 18:3 fatty acids in galactolipids, SQDG, and PG (Iba et al. 1993) (Fig. 1). First, HILIC-MS data of leaf lipids recorded in the negative ion mode were automatically extracted to yield an array of intensities of analytical signals (ions of specific $\mathrm{m} / \mathrm{z}$ value eluting at a specific time). This processing step generated lists of peak heights for 534 peaks annotated with specific LC retention times and masses (Supplementary Table 1). Listed data were then subjected to principal component analysis (PCA). The PCA model can provide an overview of all observations or samples in the data table by projecting and clustering each sample and highlighting the holistic difference of the complex metabolic state in each sample. The PCA scatter plot showed that the samples were clustered with respect to their corresponding genotypes (Fig. 7), suggesting that each mutant has a distinct metabolite profile. To identify the metabolites contributing to the separation "discriminative metabolites" between the mutant profiles and those of the wild type, for example, orthogonal projection to a latent structure-discriminant analysis (OPLS-DA) model for wild type and the ats 1-1 mutant was examined. Figure 8a shows the OPLS score scatter plot of wild type and ats $1-1$ with one predictive component. The OPLS loading S-plot, a plot of the covariance versus the correlation in conjunction with the variable trend plots allows easier visualization of the data (Fig. 8b). The variables that changed most and strongly contributed to the class separation are plotted at the top or bottom of the "S" shape plot, and those that do not vary significantly are plotted in the middle (Wiklund et al. 2008). The variables circled in Fig. $8 \mathrm{~b}$ are listed in

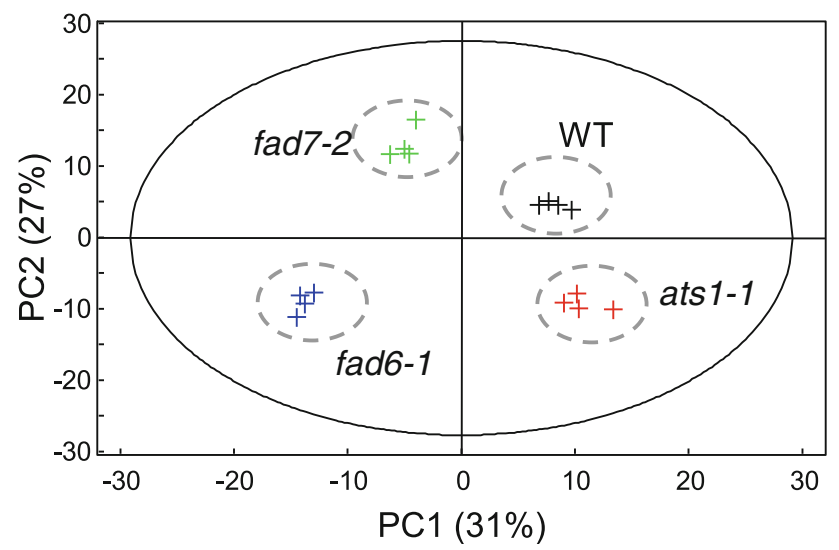

Fig. 7 Principal component analysis of ats $1-1$, fad6-1, fad7-2, and wild-type lipidome data. Principal component PC1 and PC2 represent the first 2 principal components accounting for $58 \%$ of the variance. Each plot represents an independent plant
Tables 2 and 3. The result in Table 2 showed an increase of glycoglycerolipids with two 18-carbon fatty acids in the ats $1-1$ mutant in comparison to the wild type. In addition, glycerolipids with 18-carbon fatty acid and 16-carbon fatty acid were reduced in the mutant (Table 3). These results were consistent with previous reports (Kunst et al. 1988; $\mathrm{Xu}$ et al. 2006). Lipidome data of fad6-1 and fad7-2 were also analyzed by the same procedure, and increased saturations of chloroplast glycerolipids (MGDG, DGDG, SQDG, and PG) were observed (Supplementary Figs. 5 and 6, and Supplementary Tables 2-5), also in accordance with previous reports (Iba et al. 1993; Falcone et al. 1994). These data indicate that untargeted lipidome analysis by our method can be used for detailed analysis of metabolite phenotypes.

\section{Conclusions}

We applied liquid chromatography ion trap time-of-flight mass spectrometry (LC-IT-TOF-MS) for plant lipidome
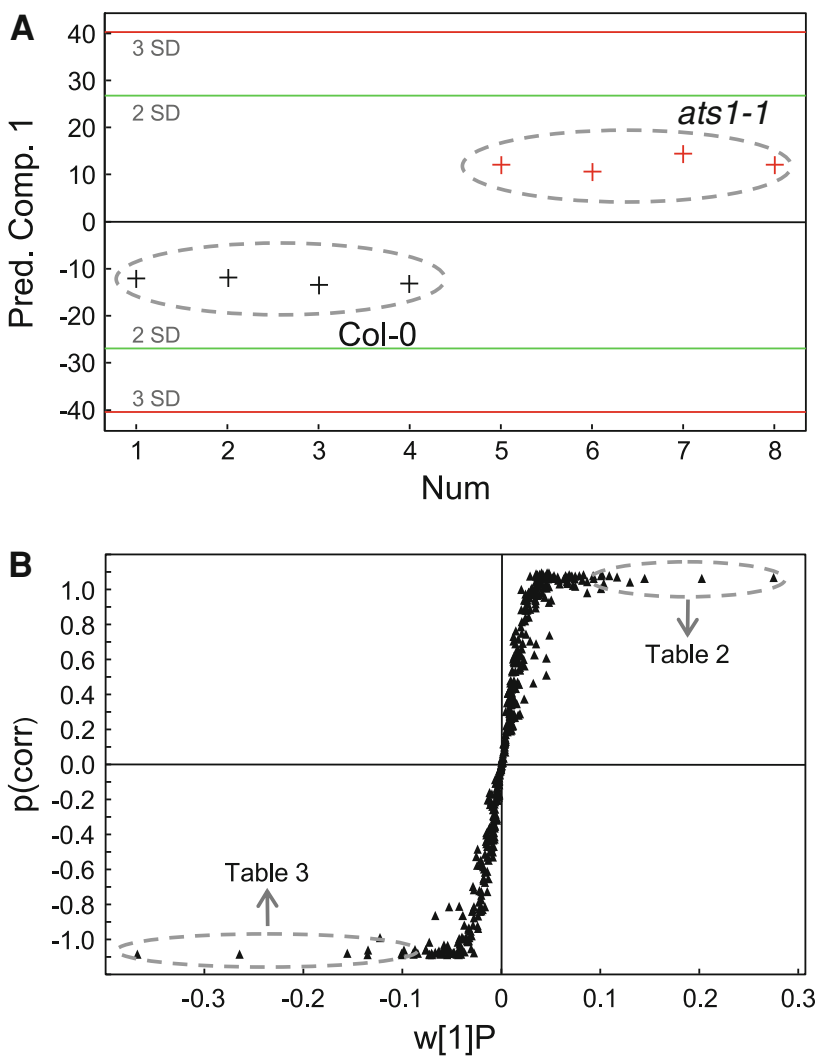

Fig. 8 OPLS-DA of ats1-1 and wild-type lipidome data. a Score scatter plot $(\mathrm{R} 2 \mathrm{Y}=0.993, \mathrm{Q} 2=0.988, \mathrm{R} 2 \mathrm{X}=0.737)$. The confidence intervals correspond to the 2 or 3 sigma limits, i.e., 2 or 3 vector standard deviations are displayed. b S-plot. The variables that changed most and strongly contributed to the class separation are circled. Details of variables circled in the upper right and lower left are shown in Tables 2 and 3 
Table 2 Discriminative metabolites whose detected levels in ats 1-1 are higher than in WT predicted by OPLS-DA
Table 3 Discriminative metabolites whose detected levels in ats 1-1 are lower than in WT as predicted by OPLS-DA

\begin{tabular}{|c|c|c|c|c|}
\hline$m / z$ & $t_{\mathrm{R}}$ & Annotation & $\begin{array}{l}\text { Fold change in peak } \\
\text { height (ats } 1-1 / \text { Col- } 0)\end{array}$ & $\mathrm{p}$ (corr) \\
\hline 819.5 & 2.49 & MGDG_36:6 $\left(\left[\mathrm{M}+\mathrm{HCO}_{2}\right]^{-}\right)$ & 2.14 & 0.975 \\
\hline 820.5 & 2.49 & MGDG_36:6 $\left(\left[\mathrm{M}+\mathrm{HCO}_{2}+1\right]^{-}\right)$ & 2.17 & 0.970 \\
\hline 821.5 & 2.47 & MGDG_36:5 $\left(\left[\mathrm{M}+\mathrm{HCO}_{2}\right]^{-}\right)$ & 2.08 & 0.971 \\
\hline 981.6 & 10.54 & DGDG_36:6 $\left(\left[\mathrm{M}+\mathrm{HCO}_{2}\right]^{-}\right)$ & 1.42 & 0.951 \\
\hline 837.5 & 9.12 & SQDG_36:6 $\left([\mathrm{M}-\mathrm{H}]^{-}\right)$ & 2.79 & 0.980 \\
\hline 823.5 & 2.45 & MGDG_36:4 $\left(\left[\mathrm{M}+\mathrm{HCO}_{2}\right]^{-}\right)$ & 3.89 & 0.986 \\
\hline 719.5 & 10.22 & PG_32:1 $\left([M-H]^{-}\right)$ & 1.66 & 0.944 \\
\hline 841.5 & 9.08 & SQDG_36:4 $\left([\mathrm{M}-\mathrm{H}]^{-}\right)$ & 10.82 & 0.991 \\
\hline 839.5 & 9.11 & SQDG_36:5 $\left([\mathrm{M}-\mathrm{H}]^{-}\right)$ & 4.03 & 0.985 \\
\hline 982.6 & 10.54 & DGDG_36:6 $\left(\left[\mathrm{M}+\mathrm{HCO}_{2}+1\right]^{-}\right)$ & 1.43 & 0.918 \\
\hline
\end{tabular}

\begin{tabular}{|c|c|c|c|c|}
\hline $\mathrm{m} / \mathrm{z}$ & $t_{\mathrm{R}}$ & Annotation & $\begin{array}{l}\text { Fold change in peak } \\
\text { height (ats } 1-1 / \text { Col- } 0)\end{array}$ & $\mathrm{p}($ corr $)$ \\
\hline 791.5 & 2.55 & MGDG_34:6 $\left(\left[\mathrm{M}+\mathrm{HCO}_{2}\right]^{-}\right)$ & 0.046 & -0.996 \\
\hline 792.5 & 2.56 & MGDG_34:6 $\left(\left[\mathrm{M}+\mathrm{HCO}_{2}+1\right]^{-}\right)$ & 0.051 & -0.996 \\
\hline 793.5 & 2.57 & MGDG_34:5 $\left(\left[\mathrm{M}+\mathrm{HCO}_{2}\right]^{-}\right)$ & 0.092 & -0.993 \\
\hline 712.5 & 12.10 & PE_34:3 $\left([\mathrm{M}-\mathrm{H}]^{-}\right)$ & 0.37 & -0.997 \\
\hline 815.5 & 9.17 & SQDG_34:3 $\left([\mathrm{M}-\mathrm{H}]^{-}\right)$ & 0.54 & -0.975 \\
\hline 781.4 & 2.64 & MGDG_34:6 $\left([\mathrm{M}+\mathrm{Cl}]^{-}\right)$ & 0.016 & -0.993 \\
\hline 800.5 & 15.70 & PC_34:3 $\left(\left[\mathrm{M}+\mathrm{HCO}_{2}\right]^{-}\right)$ & 0.38 & -0.997 \\
\hline 816.5 & 9.17 & SQDG_34:3 $\left([\mathrm{M}-\mathrm{H}+1]^{-}\right)$ & 0.51 & -0.976 \\
\hline 794.5 & 2.55 & MGDG_34:5 $\left(\left[\mathrm{M}+\mathrm{HCO}_{2}+1\right]^{-}\right)$ & 0.060 & -0.989 \\
\hline 713.5 & 12.09 & PE_34:3 $\left([\mathrm{M}-\mathrm{H}+1]^{-}\right)$ & 0.38 & -0.995 \\
\hline
\end{tabular}

analysis. Based on various authentic compounds and biological sample, our method was confirmed to be applicable to simultaneous analysis of a wide range of plant lipids containing typical membrane lipids and lipophilic secondary metabolites. Then, we applied our method for the analysis of Arabidopsis mutant $t g d 3$, and revealed an accumulation of an unusual form of DGDG $(\operatorname{Gal}(\beta 1 \rightarrow 6) \beta \mathrm{GalDG})$ and compositional differences between intact forms of $\operatorname{Gal}(\beta 1 \rightarrow 6) \beta$ GalDG, normal form of DGDG $(\operatorname{Gal}(\alpha 1 \rightarrow$ 6) $\beta$ GalDG), and MGDG which is the precursor of $\operatorname{Gal}(\beta 1 \rightarrow 6) \beta$ GalDG. Furthermore, we analyzed wellknown Arabidopsis mutants that are disrupted in lipid biosynthetic genes to examine whether untargeted lipidome analysis could be utilized for evaluation of these mutant metabolic phenotypes. The method coupled with multivariate analysis could clearly discriminate the mutants and yielded results consistent with those from previous reports. These results indicated that lipid analysis using HILIC-MS was efficient for plant lipidomics.

Acknowledgments We thank Prof. Masahiro Yano (National Institute of Agrobiological Sciences) for his kind gift of rice seeds. This work was supported in part by a grant from the Ministry of Agriculture, Forestry, and Fisheries of Japan (Genomics for
Agricultural Innovation, NVR-0005 and QTL-4010). We thank Prof. Masahiro Yano (National Institute of Agrobiological Sciences) for his kind gift of rice seeds. This work was supported in part by a grant from the Ministry of Agriculture, Forestry, and Fisheries of Japan (Genomics for Agricultural Innovation, NVR-0005 and QTL-4010).

Open Access This article is distributed under the terms of the Creative Commons Attribution Noncommercial License which permits any noncommercial use, distribution, and reproduction in any medium, provided the original author(s) and source are credited.

\section{References}

Aboshi, T., Yoshinaga, N., Nishida, R., \& Mori, N. (2010). Phospholipid biosynthesis in the gut of Spodoptera litura larvae and effects of tannic acid ingestion. Insect Biochemistry and Molecular Biology, 40(4), 325-330.

Awai, K., Xu, C., Tamot, B., \& Benning, C. (2006). A phosphatidic acid-binding protein of the chloroplast inner envelope membrane involved in lipid trafficking. Proceedings of the National Academy of Sciences of the United States of America, 103(28), 10817-10822.

Beaudoin, F., Wu, X., Li, F., Haslam, R. P., Markham, J. E., Zheng, H., et al. (2009). Functional characterization of the Arabidopsis $\beta$-ketoacyl-coenzyme A reductase candidates of the fatty acid elongase. Plant Physiology, 150(3), 1174-1191. 
Benning, C. (2009). Mechanisms of lipid transport involved in organelle biogenesis in plant cells. Annual Review of Cell and Developmental Biology, 25, 71-91.

Benning, C., \& Ohta, H. (2005). Three enzyme systems for galactoglycerolipid biosynthesis are coordinately regulated in plants. Journal of Biological Chemistry, 280(4), 2397-2400.

Bligh, E. G., \& Dyer, W. J. (1959). A rapid method of total lipid extraction and purification. Canadian Journal of Biochemistry and Physiology, 37(8), 911-917.

Browse, J., Warwick, N., Somerville, C. R., \& Slack, C. R. (1986). Fluxes through the prokaryotic and eukaryotic pathways of lipid synthesis in the '16:3' plant Arabidopsis thaliana. Biochemical Journal, 235(1), 25-31.

Brügger, B., Erben, G., Sandhoff, R., Wieland, F. T., \& Lehmann, W. D. (1997). Quantitative analysis of biological membrane lipids at the low picomole level by nano-electrospray ionization tandem mass spectrometry. Proceedings of the National Academy of Sciences of the United States of America, 94(6), 2339-2344.

Chen, M., Markham, J. E., Dietrich, C. R., Jaworski, J. G., \& Cahoon, E. B. (2008). Sphingolipid long-chain base hydroxylation is important for growth and regulation of sphingolipid content and composition in Arabidopsis. Plant Cell, 20(7), 1862-1878.

Connolly, J. D., \& Hill, R. A. (2010). Triterpenoids. Natural Product Reports, 27(1), 79-132.

Dörmann, P., Balbo, I., \& Benning, C. (1999). Arabidopsis galactolipid biosynthesis and lipid trafficking mediated by DGD1. Science, 284(5423), 2181-2184.

Falcone, D. L., Gibson, S., Lemieux, B., \& Somerville, C. (1994). Identification of a gene that complements an Arabidopsis mutant deficient in chloroplast $\omega 6$ desaturase activity. Plant Physiology, 106(4), 1453-1459.

Friedman, M. (2002). Tomato glycoalkaloids: Role in the plant and in the diet. Journal of Agricultural and Food Chemistry, 50(21), $5751-5780$.

Heinz, E., \& Roughan, P. G. (1983). Similarities and differences in lipid metabolism of chloroplasts isolated from $18: 3$ and $16: 3$ plants. Plant Physiology, 72(2), 273-279.

Hölzl, G., \& Dörmann, P. (2007). Structure and function of glycoglycerolipids in plants and bacteria. Progress in Lipid Research, 46(5), 225-243.

Houjou, T., Yamatani, K., Imagawa, M., Shimizu, T., \& Taguchi, R. (2005). A shotgun tandem mass spectrometric analysis of phospholipids with normal-phase and/or reverse-phase liquid chromatography/electrospray ionization mass spectrometry. Rapid communications in mass spectrometry, 19(5), 654-666.

Iba, K., Gibson, S., Nishiuchi, T., Fuse, T., Nishimura, M., Arondel, V., et al. (1993). A gene encoding a chloroplast $\omega-3$ fatty acid desaturase complements alterations in fatty acid desaturation and chloroplast copy number of the fad7 mutant of Arabidopsis thaliana. Journal of Biological Chemistry, 268(32), 24099-24105.

Jao, C. Y., Roth, M., Welti, R., \& Salic, A. (2009). Metabolic labeling and direct imaging of choline phospholipids in vivo. Proceedings of the National Academy of Sciences of the United States of America, 106(36), 15332-15337.

Kelly, A. A., \& Dormann, P. (2004). Green light for galactolipid trafficking. Current Opinion in Plant Biology, 7(3), 262-269.

Kelly, A. A., \& Dörmann, P. (2002). DGD2, an Arabidopsis gene encoding a UDP-galactose-dependent digalactosyldiacylglycerol synthase is expressed during growth under phosphate-limiting conditions. Journal of Biological Chemistry, 277(2), 1166-1173.

Kojima, M., Seki, K., Ohnishi, M., Ito, S., \& Fujino, Y. (1990). Structure of novel glyceroglycolipids in Adzuki bean (Vigna angularis) seeds. Biochemistry and Cell Biology, 68(1), 59-64.

Kunst, L., Browse, J., \& Somerville, C. (1988). Altered regulation of lipid biosynthesis in a mutant of Arabidopsis deficient in chloroplast glycerol-3-phosphate acyltransferase activity.
Proceedings of the National Academy of Sciences of the United States of America, 85(12), 4143-4147.

Liu, Z. Y., Huang, L. L., Chen, D. M., Dai, M. H., Tao, Y. F., Wang, Y. L., et al. (2010). Application of electrospray ionization hybrid ion trap/time-of-flight mass spectrometry in the rapid characterization of quinocetone metabolites formed in vitro. Analytical and Bioanalytical Chemistry, 396(3), 1259-1271.

Lu, B., Xu, C., Awai, K., Jones, A. D., \& Benning, C. (2007). A small ATPase protein of Arabidopsis, TGD3, involved in chloroplast lipid import. Journal of Biological Chemistry, 282(49), 35945-35953.

Malavolta, M., Bocci, F., Boselli, E., \& Frega, N. G. (2004). Normal phase liquid chromatography-electrospray ionization tandem mass spectrometry analysis of phospholipid molecular species in blood mononuclear cells: Application to cystic fibrosis. Journal of Chromatography B-Analytical Technologies in the Biomedical and Life Sciences, 810(2), 173-186.

Markham, J. E., \& Jaworski, J. G. (2007). Rapid measurement of sphingolipids from Arabidopsis thaliana by reversed-phase highperformance liquid chromatography coupled to electrospray ionization tandem mass spectrometry. Rapid Communications in Mass Spectrometry, 21(7), 1304-1314.

Markham, J. E., Li, J., Cahoon, E. B., \& Jaworski, J. G. (2006). Separation and identification of major plant sphingolipid classes from leaves. Journal of Biological Chemistry, 281(32), 22684-22694.

Michaelson, L. V., Zauner, S., Markham, J. E., Haslam, R. P., Desikan, R., Mugford, S., et al. (2009). Functional characterization of a higher plant sphingolipid $\Delta 4$-desaturase: Defining the role of sphingosine and sphingosine-1-phosphate in Arabidopsis. Plant Physiology, 149(1), 487-498.

Moellering, E. R., Muthan, B., \& Benning, C. (2010). Freezing tolerance in plants requires lipid remodeling at the outer chloroplast membrane. Science, 330(6001), 226-228.

Nakamura, Y., Koizumi, R., Shui, G., Shimojima, M., Wenk, M. R., Ito, T., et al. (2009). Arabidopsis lipins mediate eukaryotic pathway of lipid metabolism and cope critically with phosphate starvation. Proceedings of the National Academy of Sciences of the United States of America, 106(49), 20978-20983.

Okazaki, Y., Shimojima, M., Sawada, Y., Toyooka, K., Narisawa, T., Mochida, K., et al. (2009). A chloroplastic UDP-glucose pyrophosphorylase from Arabidopsis is the committed enzyme for the first step of sulfolipid biosynthesis. Plant Cell, 21(3), 892-909.

Pacetti, D., Boselli, E., Lucci, P., \& Frega, N. G. (2007). Simultaneous analysis of glycolipids and phospholids molecular species in avocado (Persea americana Mill) fruit. Journal of Chromatography A, 1150(1-2), 241-251.

Papadopoulou, K., Melton, R. E., Leggett, M., Daniels, M. J., \& Osbourn, A. E. (1999). Compromised disease resistance in saponin-deficient plants. Proceedings of the National Academy of Sciences of the United States of America, 96(22), 12923-12928.

Peters, C., Li, M., Narasimhan, R., Roth, M., Welti, R., \& Wang, X. (2010). Nonspecific phospholipase C NPC4 promotes responses to abscisic acid and tolerance to hyperosmotic stress in Arabidopsis. Plant Cell, 22(8), 2642-2659.

Rocha, J. M., Kalo, P. J., Ollilainen, V., \& Malcata, F. X. (2010). Separation and identification of neutral cereal lipids by normal phase high-performance liquid chromatography, using evaporative light-scattering and electrospray mass spectrometry for detection. Journal of Chromatography A, 1217(18), 3013-3025.

Roudier, F., Gissot, L., Beaudoin, F., Haslam, R., Michaelson, L., Marion, J., et al. (2010). Very-long-chain fatty acids are involved in polar auxin transport and developmental patterning in Arabidopsis. Plant Cell, 22(2), 364-375. 
Roughan, P. G., \& Slack, C. R. (1982). Cellular-organization of glycerolipid metabolism. Annual Review of Plant Physiology and Plant Molecular Biology, 33, 97-132.

Saito, K., \& Matsuda, F. (2010). Metabolomics for functional genomics, systems biology, and biotechnology. Annual Review of Plant Biology, 61, 463-489.

Shibano, M., Lin, A. S., Itokawa, H., \& Lee, K. H. (2007). Separation and characterization of active flavonolignans of Silybum marianum by liquid chromatography connected with hybrid ion-trap and time-of-flight mass spectrometry (LC-MS/IT-TOF). Journal of Natural Products, 70(9), 1424-1428.

Somerville, C., \& Browse, J. (1996). Dissecting desaturation: Plants prove advantageous. Trends in Cell Biology, 6(4), 148-153.

Somerville, C., Browse, J., Jaworski, J.G., \& Ohlrogge, J.B. (2000). Lipids. In Buchanan, B.B., Gruissem, W., \& Jones, R.L. (Eds.), Biochemistry \& Molecular Biology of Plants (pp. 456-527). Rockville: American Society of Plant Physiologist.

Sperling, P., \& Heinz, E. (2003). Plant sphingolipids: Structural diversity, biosynthesis, first genes and functions. Biochimica et Biophysica Acta, 1632(1-3), 1-15.

Steponkus, P. L. (1984). Role of the plasma-membrane in freezinginjury and cold-acclimation. Annual Review of Plant Physiology and Plant Molecular Biology, 35, 543-584.

Sugawara, T., \& Miyazawa, T. (1999). Separation and determination of glycolipids from edible plant sources by high-performance liquid chromatography and evaporative light-scattering detection. Lipids, 34(11), 1231-1237.

Taguchi, R., Nishijima, M., \& Shimizu, T. (2007). Basic analytical systems for lipidomics by mass spectrometry in Japan. Methods in Enzymology, 432, 185-211.

Uemura, M., Joseph, R. A., \& Steponkus, P. L. (1995). Cold acclimation of Arabidopsis thaliana (effect on plasma-membrane lipid-composition and freeze-induced lesions). Plant Physiology, 109(1), 15-30.

Welti, R., \& Wang, X. (2004). Lipid species profiling: A highthroughput approach to identify lipid compositional changes and determine the function of genes involved in lipid metabolism and signaling. Current Opinion in Plant Biology, 7(3), 337-344.

Welti, R., Wang, X., \& Williams, T. D. (2003). Electrospray ionization tandem mass spectrometry scan modes for plant chloroplast lipids. Analytical Biochemistry, 314(1), 149-152.
Wiklund, S., Johansson, E., Sjostrom, L., Mellerowicz, E. J., Edlund, U., Shockcor, J. P., et al. (2008). Visualization of GC/TOF-MSbased metabolomics data for identification of biochemically interesting compounds using OPLS class models. Analytical Chemistry, 80(1), 115-122.

Xiao, S., Gao, W., Chen, Q. F., Chan, S. W., Zheng, S. X., Ma, J., et al. (2010). Overexpression of Arabidopsis acyl-CoA binding protein ACBP3 promotes starvation-induced and age-dependent leaf senescence. Plant Cell, 22(5), 1463-1482.

Xu, C., Fan, J., Cornish, A. J., \& Benning, C. (2008). Lipid trafficking between the endoplasmic reticulum and the plastid in Arabidopsis requires the extraplastidic TGD4 protein. Plant Cell, 20(8), 2190-2204.

Xu, C., Fan, J., Froehlich, J. E., Awai, K., \& Benning, C. (2005). Mutation of the TGD1 chloroplast envelope protein affects phosphatidate metabolism in Arabidopsis. Plant Cell, 17(11), 3094-3110.

Xu, C., Fan, J., Riekhof, W., Froehlich, J. E., \& Benning, C. (2003). A permease-like protein involved in ER to thylakoid lipid transfer in Arabidopsis. EMBO Journal, 22(10), 2370-2379.

Xu, C., Yu, B., Cornish, A. J., Froehlich, J. E., \& Benning, C. (2006). Phosphatidylglycerol biosynthesis in chloroplasts of Arabidopsis mutants deficient in acyl-ACP glycerol-3-phosphate acyltransferase. Plant Journal, 47(2), 296-309.

Yunoki, K., Sato, M., Seki, K., Ohkubo, T., Tanaka, Y., \& Ohnishi, M. (2009). Simultaneous quantification of plant glyceroglycolipids including sulfoquinovosyldiacylglycerol by HPLC-ELSD with binary gradient elution. Lipids, 44(1), 77-83.

Zhang, Y., Zhu, H., Zhang, Q., Li, M., Yan, M., Wang, R., et al. (2009). Phospholipase $\mathrm{D} \alpha 1$ and phosphatidic acid regulate NADPH oxidase activity and production of reactive oxygen species in ABA-mediated stomatal closure in Arabidopsis. Plant Cell, 21(8), 2357-2377.

Zhou, J. L., Qi, L. W., \& Li, P. (2009). Herbal medicine analysis by liquid chromatography/time-of-flight mass spectrometry. Journal of Chromatography A, 1216(44), 7582-7594. 